throughout the PCC has not been described. This systematic review aims to: 1) identify and collate outcome measure (OM) definitions for the PCC stages; 2) describe how key health equity characteristics are considered in these $\mathrm{OM}$ definitions.

Methods Five databases were searched for quantitative studies published after January 1st, 2012. Data regarding study design, OM definitions, and health equity characteristics were extracted. Data were analysed using narrative synthesis.

Results 11,264 papers were identified and screened; 227 were included. The majority of studies included $>1 \mathrm{OM}$ $(67 \%)$. The most commonly reported OM was awareness (54\%), followed by interest (51\%) and uptake (50\%). Relatively few studies reported on adherence (12\%) or retention $(17 \%)$. No studies described movement through the PCC from awareness to retention. The most commonly reported equity characteristics were age (86\%) and race/ ethnicity (80\%); the least common were social capital (31\%) and religion (1\%). The majority of studies focused on cis-gender men who have sex with men (MSM) (48\%), while other affected groups, such as cis-gender women and trans* people are less well represented $(7 \%$ and $6 \%$, respectively).

Conclusion There is an unequal focus on the earlier stages of the PCC. Some key equity characteristics (e.g. age) are commonly considered, however, other important characteristics (e. g. social capital) are overlooked. These findings are relevant to healthcare professionals, policymakers and commissioners in informing how to best implement and evaluate PrEP programmes for potentially vulnerable and less advantaged populations.

\section{P184 ASSESSING INDICATOR CONDITION-GUIDED HIV TESTING IN THE HOSPITAL SETTING}

${ }^{1} S$ Bogers ${ }^{*},{ }^{1,2} \mathrm{M}$ Schim van der Loeff, ${ }^{2} \mathrm{U}$ Davidovich, ${ }^{2,3} \mathrm{~A}$ Boyd, ${ }^{1} \mathrm{M}$ van der Valk, ${ }^{4} \mathrm{~K}$ Brinkman, ${ }^{1} \mathrm{G}$ de Bree, ${ }^{1,3,5,6 \mathrm{P}}$ Reiss, $7,8 \mathrm{~J}$ van Bergen, ${ }^{1} \mathrm{~S}$ Geerlings. 'Department of Internal Medicine, Division of Infectious Diseases, Amsterdam University Medical Centers, University of Amsterdam, Amsterdam, The Netherlands; ${ }^{2}$ Department of Infectious Diseases, Public Health Service of Amsterdam, Amsterdam, The Netherlands; ${ }^{3}$ HIV Monitoring Foundation, The Netherlands; ${ }^{4}$ Department of Internal Medicine, Onze Lieve Vrouwe Gasthuis, Amsterdam, The Netherlands; ${ }^{5}$ Department of Global Health, Amsterdam University Medical Centers, University of Amsterdam, Amsterdam, The Netherlands; ${ }^{6}$ Amsterdam Institute for Global Health and Development, Amsterdam, The Netherlands; ${ }^{7}$ Department of General Practice, Amsterdam University Medical Centers, location Academic Medical Center, University of Amsterdam, Amsterdam, The Netherlands; ${ }^{8}$ STI AIDS Netherlands, Amsterdam, The Netherlands

\subsection{6/sextrans-2021-sti.278}

Background Timely diagnosis of HIV is key in the efforts to end the HIV epidemic. Indicator Condition (IC) guided HIV testing is cost-effective in identifying undiagnosed HIV. It is unknown to what extent hospitals are applying this strategy We assessed baseline findings from an ongoing multicenter interventional study aimed at identifying opportunities for improving IC-guided HIV testing in hospitals in Amsterdam.

Methods The study involved two university hospitals, two teaching hospitals, and one non-teaching hospital. Seven ICs were selected: tuberculosis (TB), lymphoma, hepatitis B
(HBV) and C (HCV), cervical carcinoma or intraepithelial neoplasia grade III (CC/CIN-III), vulvar carcinoma or intraepithelial neoplasia grade III (VC/VIN-III), and peripheral neuropathy (PN). Patients $\geq 18$ years not known to have HIV, diagnosed with one of these ICs during 2015-2020 were eligible. We included all eligible patients in one hospital, and screened a sample of $\leq 500$ patients per IC in the others. Primary outcome was the proportion of patients with an IC tested for HIV $\leq 3$ months around IC diagnosis (i.e. HIV testing ratio). Secondary outcome was the proportion of positive tests.

Results We included 4,823 patients. HIV testing ratios were highest amongst TB patients (range 74-94\%). The testing ratio varied considerably across ICs and locations, ranging between 56\%(50/90)-72\%(105/145) in lymphoma patients, $33 \%(5 / 15)-65 \%(113 / 175)$ in HBV, 29\%(2/7)-73\%(24/33) in $\mathrm{HCV}, 0 \%(0 / 68)-4 \%(16 / 452)$ in CC/CIN-III, and 4\%(4/97)$16 \%(16 / 98)$ in PN. None of the 198 VC/VIN-III patients were tested for HIV. Eleven patients (0.7\%) tested HIV positive $\leq 3$ months around IC diagnosis. Of these, 6(55\%) had lymphoma and 10(91\%) had a CD4 count $<350$ cells/ $\mathrm{mm}^{3}$.

Conclusion In-hospital IC-guided testing in the selected ICs was variably and often insufficiently practiced, but did identify people with previously undiagnosed HIV. These data show the relevance of tailored interventions to improve IC-guided HIV testing, to contribute to reducing the proportion of people with undiagnosed HIV.

\section{P188 EPIDEMIOLOGICAL AND SOCIODEMOGRAPHIC PROFILE OF HIV+/AIDS PATIENTS AT A REFERENCE CENTER IN THE SOUTH OF MINAS GERAIS, BRAZIL}

${ }^{1} V$ Pereira*, ${ }^{1} \mathrm{~T}$ de Souza, ${ }^{1,2,3,4,5} \mathrm{~A}$ Almeida. ${ }^{1}$ Medical School of Itajubá, Itajubá, Brazili ${ }^{2}$ Undergraduate Degree in Full Music Arts Education at Mozarteum College, São Paulo, Brazil; ${ }^{3}$ Master's Degree in Education from the Federal University of Goiás, Goiânia, Brazil; ${ }^{4}$ PhD in Social Sciences - History at the University of São Paulo, São Paulo, Brazil; ${ }^{5}$ PostDoctorate in the Field of Education at the Federal University of Itajubá, Itajubá, Brazil

\subsection{6/sextrans-2021-sti.279}

Introduction The Human Immunodeficiency Virus (HIV), identified in Brazil in the early 1980s, spread across the country, with its risk group and profile being modified over the years. Therefore, it is necessary to study the profile of these patients in order to generate comparisons, preventions and improvements in care. (comparisons and preventions that lead to improvements in care). Along with the dissemination, new drugs are emerging in the treatment of HIV infections, observing the emergence of comorbidities that need to be pre-diagnosed and treated, aiming at improving patients' quality of life and reducing the mortality. Given the lack of knowledge of the epidemiological and clinical profile of these patients in small and medium-sized cities, it is necessary to study and compare them at the national level. Objective

To locate the epidemiological and sociodemographic profile of patients treated at the AIDS Care and Prevention Center/ STD(CAP) in Brazil/Itajubá.

Methods Of 292 medical records of patients in 2020, 168 were randomly selected and analyzed for information such as 
sex, age, sexual orientation, viral transmission, housing, race, education, social occupation and other information on the main comorbidities.

Results There was a predominance of men (73.8\%), white $(57.7 \%)$ and $51 \%$ with incompleted high school. The predominant age groups were 31-40 years (25.6\%), 41-50 (23.8\%) and 51-60 (22.6\%). There was a predominance of heterosexual orientation $(42.3 \%)$, sexual transmission $(81.5 \%)$ and residents in the Urban Zone (81\%). In addition to Occupations: Unemployment (14.9\%), Home (14.3\%) and less favored occupants social positions. The main comorbidities were: Oral Candidiasis (35.1\%), Persistent Dermatitis (23.2\%), Depression (22\%), Pneumonia (14.3\%), Tuberculosis (13.1\%), Syphilis (10.9\%).

Conclusion The profile differs from common sense, calling attention to age and sexual orientation. Finally, there is a need to improve the filling of medical records, for future comparisons and preventions that lead to improvements in care

\section{P189 PREFERENCES OF PEOPLE LIVING WITH HIV FOR INJECTABLE AND ORAL ANTIRETROVIRAL TREATMENT IN THE NETHERLANDS: A DISCRETE CHOICE EXPERIMENT}

${ }^{1}$ I Kremer*, ${ }^{1} \mathrm{C}$ Beaudart, ${ }^{2,3}$ J Simons, ${ }^{4} \mathrm{H}$ Plieger, ${ }^{5} \mathrm{M}$ Schroeder, ${ }^{1} \mathrm{M}$ Hiligsmann. ${ }^{1}$ Maastricht University, Maastricht, The Netherlands; ${ }^{2}$ GSK, Amersfoort, The Netherlands; ${ }^{3}$ University of Groningen, University Medical Centre Groningen, Groningen, The Netherlands; ${ }^{4}$ ViV Healthcare, Amersfoort, The Netherlands; ${ }^{5}$ ViiV HealthCare, London, UK

\subsection{6/sextrans-2021-sti.280}

Background Antiretroviral (ARV) therapies are currently orally administered, daily; however, there are long-acting (LA) injectable alternative ARVs in development. Understanding preferences of people living with HIV (PLWH) for different modes of administration, could provide relevant information for clinical and policy decision-making. This study aimed to elicit preferences of PLWH for LA injectable- compared with oral ARVtherapies, in the Netherlands.

Methods A discrete-choice experiment (DCE) was conducted among PLWH, which presented 12 choice tasks for two ARV therapies (i.e. oral or LA injectable). PLWH were asked in each task to select their preferred ART. Based on literature reviews, interviews with PLWH and expert consultation, ARV options were characterized by six attributes: location of administration, dosing frequency, risk of having short-term side effects, risk of drug-drug interaction, forgivability, and food and mealtime restrictions. Random parameters logit and latent class models were used to estimate preferences of PLWH.

Results A total of 76 PLWH completed the survey. Of the 76 respondents, $22(28.9 \%)$ chose oral ARV therapy in all choice tasks, $30(39.5 \%)$ respondents always chose LA injectable ARV therapy, and $24(31.6 \%)$ respondents varied their choice depending on the choice tasks. Respondents always choosing LA injection reported to be more willing to switch their ARV therapy $(\mathrm{p}=.006)$. On average, PLWH preferred an injectable therapy, a less frequent dosing regimen, lower risks of side effects or drug-drug interactions, therapy administrated at home and therapy without food and mealtime restrictions, compared with alternatives.

Conclusion This study revealed that PLWH have a strong preference for mode of administration. Some people always preferred LA injectable ARV therapy, while others always preferred oral ARVs. This study, therefore, highlights the value and potential marketplace for a LA injectable ARV therapy. Improved medication adherence could result, if patients are actively involved in the decision-making process of their ARV treatment.

\section{P190 DECLINING TRENDS OF SYPHILIS SEROPREVALENCE IN INDIA: A 10-YEAR-ANALYSIS}

P Srivastava*, A Bhargava, S Bansal, N Khunger. Vardhman Mahavir Medical College and Safdarjung Hospital, New Delhi, India, New Delhi, India

\subsection{6/sextrans-2021-sti.281}

Background World Health Organization (WHO) estimates that each year 11 million new cases of syphilis occur globally. Syphilis remains an important public health challenge in lowincome countries like India and has re-emerged in several high-income countries. The present study was undertaken to analyse the trends in prevalence, pattern and clinical presentations of syphilis, over 10 years, at a tertiary-care-hospital in India.

Methods A retrospective observational study was carried out at the Apex Regional STD Centre, Safdarjung Hospital, New Delhi, India; wherein data from patient records of all the syphilis cases from sexually transmitted infections (STIs) clinic, from January 2007 to December 2016 was analysed. Patients were diagnosed using syphilis serological assays as per classical testing strategy. Epidemiological, clinical and investigational data was analysed statistically for changing epidemiological and clinical trends of syphilis.

Results Of 373,845 patients tested for syphilis, $1.41 \%$ males were VDRL reactive compared to $0.49 \%$ females $(\mathrm{x} 2=874.5$, $\mathrm{p}<0.0001)$. VDRL reactivity showed a declining trend with $1.56 \%$ prevalence in 2007 and $1.05 \%$ in 2016 ( $\mathrm{x} 2$ trend $=87.8, \mathrm{p}<0.0001)$ with significant fall in reactivity for females. TPHA reactivity also showed a declining trend $(\mathrm{x} 2$ trend $=32.0, \mathrm{p}<0.0001)$. Biological false positivity was appreciably more in females $(\mathrm{x} 2=4.06, \mathrm{p}=0.043)$ and with VDRL titres $<1: 8(\mathrm{x} 2=44.1, \mathrm{p}<0.0001)$. TPHA positivity was significantly higher with VDRL titres $\geq 1: 8 \quad(\mathrm{x} 2=55.2$, $\mathrm{p}<0.0001)$. Latent syphilis comprised $92.7 \%$ of total diagnosed cases $(p<0.0001)$ and $20-29$ years was the most common age-group with $55.8 \%$ cases $(\mathrm{p}<0.0001)$.

Conclusion Syphilis has features of a disease that could be eliminated; like absence of reservoir, 2-6 week incubation period, availability of inexpensive sero-diagnostic tests and treatable with single dose of penicillin. Since, syphilis has been recommended as a marker of STI prevalence by WHO, the strong and significant decline in seroprevalence of syphilis during 2007-2016, reflects the success of the National STI Prevention and Control Programme whose pillars rest upon education and proper management. 